\title{
Detection of carbapenem resistant Klebsiella species isolated from clinical samples
}

\author{
Sakina Yousuf Ali and Taqdees Malik* \\ Department of Microbiology, Jinnah University for Women, Karachi-Pakistan \\ *Corresponding author's email: taqdees_13@ hotmail.com \\ Citation \\ Sakina Yousuf Ali and Taqdees Malik. Detection of carbapenem resistant Klebsiella species isolated from \\ clinical samples. Pure and Applied Biology. Vol. 9, Issue 2, pp1662-1666. \\ http://dx.doi.org/10.19045/bspab.2020.90175
}

\begin{tabular}{llll}
\hline \hline Received: 07/01/2020 & Revised: 20/03/2020 & Accepted: 22/03/2020 & Online First: 24/03/2020 \\
\hline \hline
\end{tabular}

\section{Abstract}

The dissemination of carbapenem resistance among the family of Enterobacteriaceae is one of the most potent intimidation to an individual's health nowadays. In this research, the foremost focus is on carbapenemase-producing Klebsiella species. Carbapenems are a genre of betalactam ring containing antibiotics which includes meropenem imipenem, ertapenem, biapenem, doripenem \& panipenem. Carbapenems are highly effective antibiotics as they arrest the cell wall of bacteria so if the resistance against carbapenem emerges than it will subsequently lead to narrow therapeutic options. The foremost purpose of this research is the determination of carbapenem resistance among Klebsiella species. Fifty $(\mathrm{n}=50)$ gram-negative samples were collected from the Centralized Diagnostic Laboratory of Karachi, Pakistan. All the samples were sub-cultured on EMB (eosin methylene blue) agar to differentiate them from other Enterobacteriaceaes. MacConkey agar was used to check lactose fermentation. Gram staining and capsule staining for morphological identification, biochemical tests (Indole, Methyl red, Voges Proskauer, Citrate, (IMViC), Triple Sugar Iron (TSI)) were carried out to differentiate among Klebsiella species. Carbapenem resistance against Imipenem and Meropenem was identified by the disc diffusion method based on the Clinical Laboratory Standard Institute (CLSI) guidelines. Out of which 26 isolates were identified as Klebsiella spp. In these 26 isolates, 10 isolates were identified as Klebsiella pneumoniae. Further results showed that $K$. pneumoniae isolates were more resistant to Meropenem as compared to the Imipenem. All ten isolates of K. pneumoniae were found resistant to Meropenem while only two isolates of $K$. pneumoniae were resistant to Imipenem. This study was done to determine the carbapenem resistance among Klebsiella so that data can be provided which will help in rapid and appropriate treatment of patients so that in the future we can use alternative drugs or cocktail of drugs to treat infections caused by Klebsiella.

Keywords: Beta-lactamases; Carbapenems; Emergence; Klebsiella pneumoniae; Resistance

\section{Introduction}

The Genus Klebsiella consists of gram -ve rods, capsulated, 1-2 micrometer long and non-motile organisms, favorably grew in a moist environment. They are facultative anaerobes. The Genus Klebsiella associated with the family of Enterobacteriaceae [1,
2]. Klebsiella is amidst the foremost probable elements of a spread of society and healthcare-associated infections. Healthcare-associated diseases of Klebsiella cannot be easily discriminated medically from other microorganism involved in different diseases. However, 
society-associated diseases of Klebsiella may have some indicative options [1]. Historically, Genus Klebsiella has been thought to be a very important reason for community-acquired respiratory disorders. Klebsiella pneumoniae followed by Klebsiella oxytoca is the most prominent species of the genus, found to be associated with human clinical specimens [1].

Nowadays the major global problem found is antibiotic resistance or antimicrobial resistance (AMR). The extrajudicial use of antibacterial agents has led to antimicrobial resistance both in pathogenic and commensal organisms [3]. There are two possible ways through which drug resistance can develop, one is horizontal gene transfer and the other is dissimilar chromosomal loci through mutations [4].

$K$. pneumoniae have two major antibiotic resistance mechanism in them. One pathway involves the expression of extended-spectrum $\beta$-lactamases (ESBLs) that contributes to produce resistance in $K$. pneumoniae against cephalosporin and monobactam. Another extremely worse resistance mechanism is that the expression of Carbapenemases by $K$. pneumoniae, which contributes to the resistance of $K$. pneumoniae against most offered $\beta$-lactams as well as the carbapenems [5].

The foremost report of $K$. pneumoniae presenting a carbapenemase was known in 1996 in the North geographical area \& therefore, this sort of carbapenemase is termed as $K$. pneumoniae carbapenemase (KPC) [6]. Carbapenemases present the adjustable clan of $\beta$-lactamases with a wider spectrum unequaled by different beta-lactam-degrading enzymes. Researchers have given the terminology "carbapenem-hydrolyzing enzymes" to the term "carbapenemases" [7]. Carbapenemases are particular betalactamases with the flexibility to degrade carbapenems [8].

Carbapenems come under the class of Betalactam antibiotics that attach to penicillinbinding proteins thus constraining the synthesis of the outer cellular wall that leads to bacterial killing [9]. Carbapenems have a fully wider spectrum. For Grampositive micro-organisms, effective antibiotics are doripenem, imipenem and panipenem. For Gram-negative microorganisms, potent antibiotics are meropenem, ertapenem, doripenem and biapenem [10]. Resistance mechanism against carbapenems in $K$. pneumoniae occurs due to betalactamases/carbapenemase production, alteration in the expression or function of Penicillin Binding Proteins (PBPs) or porins due to mutations and efflux pump system. Together these mechanisms can lead to a high level of resistance against Carbapenems among K. pneumoniae [11].

In this research, the main focus was to detect Carbapenem resistance among $K$. pneumoniae which is due to the expression of Carbapenemases. So, that spread of resistant strains can be restricted with proper therapy and effective drugs.

\section{Material and methods \\ Specimen collection}

Fifty $(n=50)$ gram-negative samples were collected from the Centralized Diagnostic Laboratory of Karachi, Pakistan. All the isolates were aseptically streaked on MacConkey agar and EMB agar. Identification and characterization of bacterial isolates were performed by using standard microbiological techniques.

Antibiotic susceptibility checking (By disc diffusion-kirby-bauer technique)

This technique is one of the most used and efficient in microbiology for antibiotic susceptibility checking. In this test, a suspension of inoculum is prepared and then compared to the McFarland standard. Inoculum is then spread evenly on the Mueller Hinton Agar (MHA). Small filter paper discs impregnated with specific antibiotics are then placed onto an agar plate to which bacteria have been spread. Plates are incubated overnight at $37^{\circ} \mathrm{C}$. Inhibition zone following the impregnated discs is measured on the next day according to CLSI guidelines. Zone of inhibition can state either the microorganism is sensitive 
(S), intermediate or resistant $(\mathrm{R})$ to the given antibiotics. The commercially prepared antibiotic disc used for the samples of Klebsiella species were: Imipenem (10ug) and Meropenem (10ug) (both belonging to Carbapenem family).

\section{Results}

Out of 50 Gram-negative isolates, 26 isolates were identified as Klebsiella spp. In these 26 isolates, 10 isolates were identified as Klebsiella pneumoniae by using standard microbiological techniques According to zone of inhibitions showed in (Fig. 1), K. pneumoniae were found highly resistant to Meropenem as compared to Imipenem. All ten isolates of $K$. pneumoniae were resistant to Meropenem whereas two isolates of $K$. pneumoniae showed resistance against Imipenem and the rest were sensitive to Imipenem as mentioned in the (Table 1).

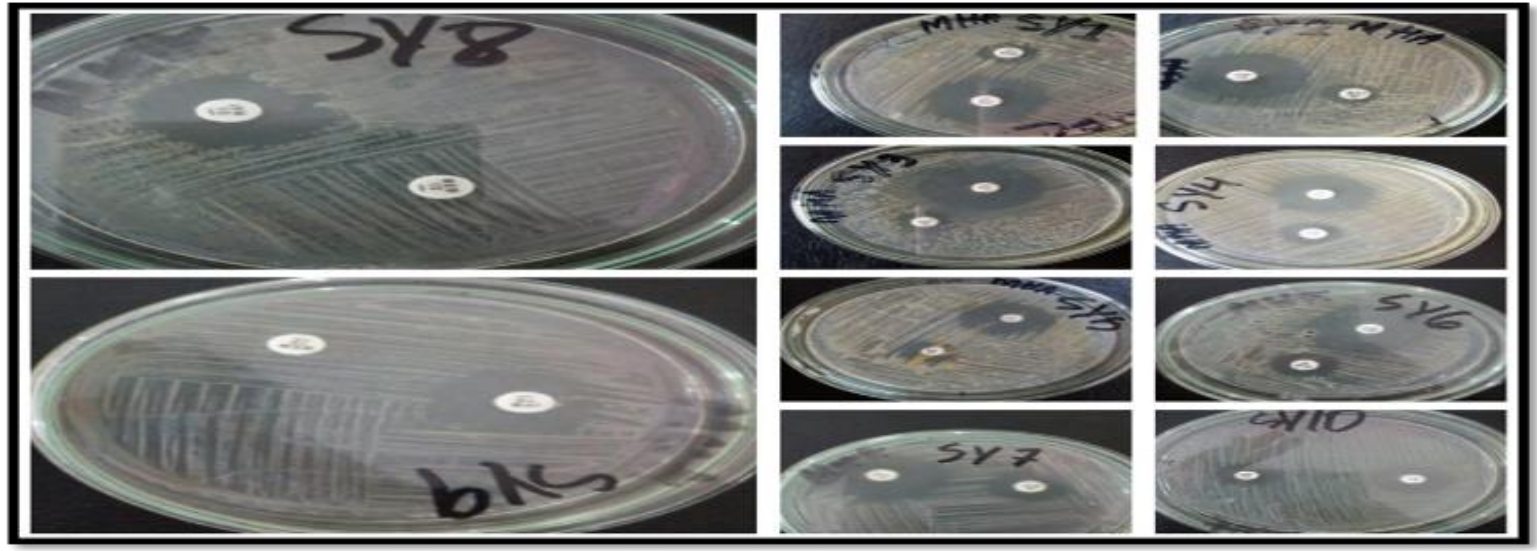

Figure 1. Antibiotic susceptibility testing performed by disc diffusion-Kirby Bauer technique shows different zones of inhibition of Imipenem and Meropenem against Klebsiella pneumoniae

Table 1. Antibiotic susceptibility test performed by disc diffusion-Kirby Bauer technique as shown in figures 1(a-c)

\begin{tabular}{|c|c|c|c|}
\hline Organisms & Antibiotic disc (10UG) & Zone of inhibitions (MM) & Interpretation a/c to clsi data \\
\hline Sy1 & Imipenem & $25 \mathrm{~mm}$ & Sensitive \\
\hline Sy2 & Imipenem & $30 \mathrm{~mm}$ & Sensitive \\
\hline Sy3 & Imipenem & $31 \mathrm{~mm}$ & Sensitive \\
\hline Sy4 & Imipenem & $25 \mathrm{~mm}$ & Sensitive \\
\hline Sy5 & Imipenem & $27 \mathrm{~mm}$ & Sensitive \\
\hline Sy6 & Imipenem & $31 \mathrm{~mm}$ & Sensitive \\
\hline Sy7 & Imipenem & $30 \mathrm{~mm}$ & Sensitive \\
\hline Sy8 & Imipenem & $18 \mathrm{~mm}$ & Resistant \\
\hline Sy9 & Imipenem & $17 \mathrm{~mm}$ & Resistant \\
\hline Sy10 & Imipenem & $27 \mathrm{~mm}$ & Sensitive \\
\hline Sy1 & Meropenem & $13 \mathrm{~mm}$ & Resistant \\
\hline Sy2 & Meropenem & $13 \mathrm{~mm}$ & Resistant \\
\hline Sy3 & Meropenem & $12 \mathrm{~mm}$ & Resistant \\
\hline Sy4 & Meropenem & $15 \mathrm{~mm}$ & Resistant \\
\hline Sy5 & Meropenem & $19 \mathrm{~mm}$ & Resistant \\
\hline Sy6 & Meropenem & $16 \mathrm{~mm}$ & Resistant \\
\hline Sy7 & Meropenem & $15 \mathrm{~mm}$ & Resistant \\
\hline Sy8 & Meropenem & No zone & Resistant \\
\hline Sy9 & Meropenem & No zone & Resistant \\
\hline Sy10 & Meropenem & $19 \mathrm{~mm}$ & Resistant \\
\hline
\end{tabular}




\section{Discussion}

Nowadays one of the major issues is antibiotic resistance amongst microorganisms. Resistance against carbapenems mainly amongst Gram -ve microorganism is an ongoing public challenge for the globe. Beta lactams are the maximum used antibiotics internationally, consist of Penicillin, Cephalosporin, Monobactams, and Carbapenems. They all have a common beta-lactam ring and for this reason, they are capable of inactivating penicillinbinding proteins that are an important function for the bacterial wall [12]. Carbapenems are the beta-lactams that can be used for to Gram -ve and Gram +ve bacteria presenting a vast spectrum of antibacterial activity. Carbapenems are frequently given as a final therapeutic choice when sufferers with infections grow to be severely unwell or they're suspected of harboring resistant pathogens. Unfortunately, nowadays the emergence of multi-drug resistant (MDR) pathogens seriously threatens this class of life-saving antibiotics. Several latest studies have shown that resistance to carbapenems is increasing at some stage in the world [6]. Carbapenem-resistant in K. Pneumoniae is in particular due to the expression of carbapenemases. Other mechanisms of resistance to carbapenems are the manufacturing of beta-lactamases, efflux pumps, and mutations that modify the expression or function of porins and Penicillin-binding proteins. Combinations of those mechanisms can cause excessive ranges of resistance to carbapenems in certain bacterial species which include $K$. Pneumoniae [13]. Notwithstanding the type of carbapenemase that is carried, carbapenem-resistant $K$. Pneumoniae segregation is called CRE for carbapenemresistant Enterobacteriaceae [5]. In this research, the primary attention turned into carbapenem-resistant K. Pneumoniae, for this motive Imipenem and Meropenem (both belong to the carbapenem class) were used. Results showed that K. Pneumoniae is relatively more resistant against Meropenem as compared to Imipenem. Similar to our study, it was found in a research that all 38 experimental isolates have been resistant to beta-lactam antibiotics in which 37 strains out of 38 experimental isolates were resistant to imipenem which was also confirmed by genotypic characterization of carbapenemase gene. [14].

\section{Conclusion}

In this research, it was found that $K$. pneumoniae were highly resistant to Meropenem and less to Imipenem. Thus, it is concluded that the emergence of resistance against carbapenems due to production of carbapenemases is increasing day by day and it is now a major concern for public health.

\section{Authors' contributions}

Conceived and designed the experiments: $\mathrm{T}$ Malik, Performed the experiments: SY Ali, Analyzed the data: T Malik \& SY Ali, Contributed reagents/ materials/ analysis tools: T Malik \& SY Ali, Wrote the paper: SY Ali.

\section{References}

1. Paterson DL, Siu KL \& Chang FY (2018). Klebsiella Species: (K. Pneumoniae, K. Oxytoca, K. Ozaenae and $K$. Rhinoscleromatis). Infect Dis \& Antimicrob Agents 64(3).

2. Jin Y, Song X, Liu Y, Wang Y, Zhang B, Fan H \& Shao C (2017). Characteristics of CarbapenemaseProducing Klebsiella Pneumoniae as a Cause of Neonatal Infection in Shandong, China, Exp Ther Med 13(3): 1117-1126.

3. Munita JM \& Arias CA (2015). Mechanisms of Antibiotic Resistance, Microbiol Spect 4(2): 10.1128.

4. Aminov RI \& Mackie RI (2007). Evolution and Ecology of Antibiotic Resistance Genes, FEMS Microbiol Lett 271(2): 147-161.

5. Queenan AM \& Bush K (2007). Carbapenemases: The Versatile BLactamases, Clin Microbiol Rev 20(3): 440-458. 
6. Papp-Wallace KM, Endimiani A, Taracila MA \& Bonomo RA (2011). Carbapenems: Past, Present, and Future, Antimicrob Agents Chemother 55(11): 4943-4960.

7. Chen L, Mathema B, Chavda KD, Deleo FR, Bonomo RA \& Kreiswirth BN (2014). Carbapenemase-Producing Klebsiella Pneumoniae: Molecular and Genetic Decoding. Trends Microbiol 22(12): 686-696.

8. Munoz-Price LS, Poirel L, Bonomo RA, Schwaber MJ, Daikos GI, Cormican M, Cornaglia G, Garau J, Gniadkowski M \& Hayden MK et al. (2013). Clinical Epidemiology of the Global Expansion of Klebsiella Pneumoniae Carbapenemases. Lancet Infect Dis 13: 785-796.

9. Nicolau DP (2008). Carbapenems: A Potent Class of Antibiotics. Expert Opin Pharmacother 9(1): 23-37.

10. Zhanel GG, Wiebe R, Dilay L, Thomson K, Rubinstein E, Hoban DJ, Noreddin AM \& Karlowsky JA (2007).
Comparative Review of The Carbapenems. Drugs 67(7): 1027-52.

11. El-Gamal MI, Brahim I, Hisham N, Aladdin R, Mohammed $\mathrm{H}$ \& Bahaaeldin A (2017). Recent Updates of Carbapenem Antibiotics, Eur J Med Chem 131: 185-195.

12. Meletis G (2016). Carbapenem Resistance: Overview of The Problem and Future Perspectives, Ther Adv Infect Dis 3(1): 15-21.

13. Mena A, Plasencia V, García L, Hidalgo O, Ayestarán JI, Alberti S, Borrell N, Pérez JL \& Oliver A (2006). Characterization of A Large Outbreak By CTX-M-1-Producing Klebsiella Pneumoniae \& Mechanisms Leading To In Vivo Carbapenem Resistance Development. J Clin Microbiol 44(8): 2831-2837

14. Lin D, Chen J, Yang Y, Cheng J \& Sun C (2018). Epidemiological Study of Carbapenem-resistant Klebsiella Pneumoniae. Open Med (Wars) 13: 460-466. 